\title{
SUPERDIAGONAL FORMS FOR COMPLETELY CONTINUOUS LINEAR OPERATORS
}

\author{
by DEMETRIOS KOROS
}

(Received 22 February, 1981)

\begin{abstract}
Altman [1] showed that Riesz-Schauder theory remains valid for a completely continuous linear operator on a locally convex Hausdorff topological vector space over the complex field. In a later paper [2], he proved an analogue of the Aronszajn-Smith result; specifically, he showed that such an operator possesses a proper closed invariant subspace. The purpose of this paper is to show that Ringrose's theory of superdiagonal forms for compact linear operators [3] can be generalized to the case of a completely continuous linear operator on a locally convex Hausdorff topological vector space over the complex field. However, the proof given in [3] requires considerable modification.
\end{abstract}

Terminology pertaining to locally convex Hausdorff topological vector spaces in this paper is as in the book [5]. Throughout, $X$ is a fixed locally convex Hausdorff topological vector space over the complex field and $X \neq\{0\}$.

Definition. A linear operator $T$ on $X$ is called completely continuous if there is a neighbourhood $U$ of $O$ such that $T U$ is a precompact set.

We observe that, by Proposition 7.2 of [5; p. 58], the topology of $X$ has a basis of barrels. Since any subset of a precompact set is precompact, we may assume without loss of generality that $U$ is a barrel.

Next, we describe Altman's generalization of Riesz-Schauder theory [1].

THEOREM A. Let $T$ be a completely continuous linear operator on $X$, and let $\lambda$ be a non-zero complex number. There are two possibilities:

(a) $\lambda I-T$ is a homeomorphism of $X$ onto itself;

(b) $\lambda$ is an eigenvalue of $T$.

The set of points which satisfy $(b)$ is countable and it has no cluster point except possibly zero. Let $\lambda$ be a non-zero eigenvalue of T. Then there is a positive integer $\nu(\lambda)$ with the following properties.

(i) For each positive integer $n,(\lambda I-T)^{n} X$ is closed. Also

$$
(\lambda I-T)^{m+1} X=(\lambda I-T)^{m} X \quad(m \geq \nu(\lambda))
$$

and $\nu(\lambda)$ is the smallest positive integer with this property.

(ii) For each positive integer $n, N\left((\lambda I-T)^{n}\right)$, the null-space of $(\lambda I-T)^{n}$, is finitedimensional. Also

$$
N\left((\lambda I-T)^{m}\right)=N\left((\lambda I-T)^{m+1}\right) \quad(m \geq \nu(\lambda))
$$

and $\nu(\lambda)$ is the smallest positive integer with this property.

(iii) $(\lambda I-T)^{m} X \oplus N\left((\lambda I-T)^{m}\right)=X \quad(m \geq \nu(\lambda))$.

(iv) If $d(\lambda)$ is the dimension of the null-space of $(\lambda I-T)^{\nu(\lambda)}$, then

$$
1 \leqslant \nu(\lambda) \leqslant d(\lambda) \text {. }
$$

Glasgow Math. J. 23 (1982) 163-170. 
NOTE. The integers $\nu(\lambda)$ and $d(\lambda)$ are called respectively the index and the algebraic multiplicity of the eigenvalue $\lambda$.

Altman [2] proved the following result.

THEOREM B. Let $T$ be a completely continuous linear operator on $X$. Then there is $a$ proper closed subspace $Y$ of $X$ invariant under $T$, provided only that $X$ has dimension at least two.

Prior to proving the main results of this paper we require a preliminary result on quotient spaces.

THEOREM C. Let $M$ be a closed linear subspace of $X$. Let $\phi$ be the canonical mapping of $X$ onto $X / M$. Then

(i) the topology of $X / M$ is locally convex;

(ii) if $P$ is a basis of continuous seminorms on $X$, we denote by $P_{M}$ the family of seminorms on $X / M$ consisting of the seminorms

$$
[x] \rightarrow \tilde{p}([x])=\inf \{p(y): y \in[x]\} ;
$$

then $P_{M}$ is a basis of continuous seminorms on $X / M$;

(iii) $\phi$ is a continuous mapping of $X$ onto $X / M$.

For a proof of this result, the reader is referred to Proposition 7.9 of [5, p. 65]. Whenever, in this chapter, a quotient space is introduced it will be assumed that it has been topologised in the manner specified above.

Throughout the remainder of this paper, $T$ denotes a fixed completely continuous linear operator on $X$. The term subspace will be used to describe a closed linear subspace of $X$. Clearly if $Y$ is an invariant subspace for $T$, then $T \mid Y$, the restriction of $T$ to $Y$, is also completely continuous. We have also the following result.

PROPOSITION 1. Let $Y$ be an invariant subspace for $T$. Then the operator $T_{Y}$ defined on the quotent $X / Y$ by

$$
T_{Y}[x]=[T x] \quad(x \in X)
$$

is a completely continuous operator.

Proof. By definition there is a neighbourhood $U$ of 0 such that the image $T U$ is precompact. Let $\phi$ be the canonical mapping of $X$ onto $X / Y$. Then $\phi(U)$ is a neighbourhood of the zero element in $X / Y$ and, moreover, $T_{Y} \phi(U)=\phi(T U)$. By [4, p. 49], the continuous image of a precompact set is precompact. It follows that $T_{Y}$ is completely continuous.

A family $\mathscr{F}$ of subspaces of $X$, which is totally ordered by the inclusion relation, will be termed a nest of subspaces. If in addition each subspace in $\mathscr{F}$ is invariant under $T$ we shall describe $\mathscr{F}$ as an invariant nest. A trivial example of an invariant nest is the family consisting of the two subspaces $\{0\}, X$. Non-trivial invariant nests may be constructed using Altman's result, Theorem B.

We shall use the symbol $\subseteq$ to denote the inclusion relation and reserve $\subset$ for strict inclusion. The strong closure of a subset $S$ of $X$ will be denoted by cl $S$. Given a nest $\mathscr{F}$ of 
subspaces of $X$ and $M \in \mathscr{F}$, we define

$$
M_{-}=\operatorname{cl}[\bigcup\{L: L \in \mathscr{F}, L \subset M\}] .
$$

If there is no $L$ in $\mathscr{F}$ such that $L \subset M$, we define $M_{-}=\{0\}$. It is clear that $M_{-}$is a subspace of $X$, and that it will be an invariant subspace if $\mathscr{F}$ is an invariant nest. Also $M_{-} \subseteq M$. It should be emphasized that the definition of $M_{-}$depends on the particular nest $\mathscr{F}$ under consideration and not merely on the subspace $M$. We shall say that $\mathscr{F}$ is continuous at $M$ if $M=M_{-}$.

A nest $\mathscr{F}$ will be termed simple if

(i) $\{0\} \in \mathscr{F}, X \in \mathscr{F}$;

(ii) if $\mathscr{F}_{0}$ is any subfamily of $\mathscr{F}$, then the subspaces

$$
\bigcap\left\{L: L \in \mathscr{F}_{0}\right\}, \operatorname{cl}\left[\bigcup\left\{L: L \in \mathscr{F}_{0}\right\}\right]
$$

are in $\mathscr{F}$;

(iii) if $M \in \mathscr{F}$, then the quotient space $M / M_{-}$is at most one-dimensional.

We note that condition (ii) implies that $M_{-} \in \mathscr{F}$ whenever $M \in \mathscr{F}$.

TheOREM 1. There exists a simple nest $\mathscr{F}$, each of whose members is a subspace invariant under $T$.

Proof. Let $\mathcal{N}_{i}$ denote the class of all invariant nests. Then $\mathcal{N}_{i}$ is not empty since it contains the trivial nest consisting of the subspaces $\{0\}, X$. The class $\mathcal{N}_{i}$ may be partially ordered by inclusion; if $\mathscr{F}_{1}, \mathscr{F}_{2} \in \mathcal{N}_{i}$, we say $\mathscr{F}_{1}<\mathscr{F}_{2}$ if every subspace in the family $\mathscr{F}_{1}$ is also member of $\mathscr{F}_{2}$. It is easily seen that, in this way, $\mathcal{N}_{i}$ is inductively ordered; for if $\mathcal{N}_{0} \subseteq \mathcal{N}_{i}$ and $\mathcal{N}_{0}$ is totally ordered by the relation $<$, then

$$
\mathscr{F}_{0}=\bigcup\left\{\mathscr{F}: \mathscr{F} \in \mathcal{N}_{0}\right\}
$$

is the least upper bound of $\mathcal{N}_{0}$ in $\mathcal{N}_{i}$. We may now deduce from Zorn's lemma the existence of at least one maximal nest of invariant subspaces, $\mathscr{F}$ say.

It is apparent that $\{0\}, X \in \mathscr{F}$, since otherwise $\mathscr{F}$ could be enlarged by the addition of these subspaces, contrary to the assumption that $\mathscr{F}$ is maximal. Moreover, let $\mathscr{F}_{0}$ be a sub-family of $\mathscr{F}$ and consider

$$
M_{0}=\bigcap\left\{L: L \in \mathscr{F}_{0}\right\} .
$$

It is evident that that $M_{0}$ is a closed subspace of $X$. Let $M \in \mathscr{F}$. Since $\mathscr{F}$ is totally ordered by inclusion we have either (a) $M \subseteq L\left(L \in \mathscr{F}_{0}\right)$ and $M \subseteq M_{0}$, or (b) $L \subset M$ for some $L$ in $\mathscr{F}_{0}$ and $M_{0} \subset M$. It follows that the family obtained by adding $M_{0}$ to $\mathscr{F}$ remains totally ordered by inclusion and is therefore an invariant nest. Since $F$ is maximal we deduce that $M_{0} \in \mathscr{F}$. A similar argument shows that

$$
\operatorname{cl}\left[\bigcup\left\{L: L \in \mathscr{F}_{0}\right\}\right] \in \mathscr{F} \text {. }
$$

Hence, in order to prove that $\mathscr{F}$ is a simple invariant nest for $T$ it remains to verify that, given any $M$ in $\mathscr{F}$, the quotient space $M / M_{-}$is at most one-dimensional. Suppose that, for some $M$ in $\mathscr{F}$, this is not the case. When $x \in M$ we denote by $[x]$ the coset 
$x+M_{-}$. It follows from results stated earlier that $M / M_{-}$is a locally convex Hausdorff topological vector space. Since $M$ and $M_{-}$are invariant under $T$, we may define a linear operator $T_{M}$ from $M / M_{-}$into itself by the equation

$$
T_{M}[x]=[T x] \quad(x \in M) .
$$

It follows from Proposition 1 that $T_{M}$ is a completely continuous linear operator. Since $M / M_{-}$has dimension exceeding one, Theorem $B$ implies the existence of a proper subspace $L_{M}$ of $M / M_{-}$which is invariant under $T_{M}$. If we now put

$$
L=\left\{x: x \in M,[x] \in L_{M}\right\},
$$

then $L$ is a subspace of $X$, being the inverse image under the continuous linear map $x \rightarrow[x]$ of the subspace $L_{M}$, such that $M_{-} \subset L \subset M$. Given any subspace $N$ in $\mathscr{F}$ we have either (a) $M \subseteq N$ and $L \subset N$ or (b) $N \subset M$ and

$$
N \subseteq \mathrm{cl}[\bigcup\{K \in \mathscr{F}: K \subset M\}]=M_{-} \subset L .
$$

It follows that $L \notin \mathscr{F}$, and that the family obtained by adding $L$ to $\mathscr{F}$ is a nest. This contradicts the assumption that $\mathscr{F}$ is maximal. Hence $M / M_{-}$is at most one-dimensional, for every $M$ in $\mathscr{F}$, and so $\mathscr{F}$ is a simple nest.

Throughout the remainder of this paper we shall use the symbols $T, \mathscr{F}$ with the meanings attributed to them in the statement of Theorem 1 . If $M \in \mathscr{F}$, then either $M=M$. or $M / M$ has dimension one. In the latter case let $z_{M} \in M \backslash M_{-}$. Then, since $M$ is invariant under $T$, we have $T z_{M} \in M$, and hence $T z_{M}$ can be expressed uniquely in the form

$$
T z_{M}=\alpha_{M} z_{M}+y_{M}
$$

where $\alpha_{M}$ is a scalar and $y_{M} \in M_{-}$. It is easily verified that $\alpha_{M}$ does not depend on the particular choice of $z_{M}$. When $M=M_{-}$we define $\alpha_{M}=0$. In this way we associate with each $M$ in $\mathscr{F}$ a scalar $\alpha_{M}$ which we shall call the diagonal coefficient of $T$ at $M$.

Let $\alpha$ be a scalar. We define the diagonal multiplicity of $\alpha$ to be the number (possibly infinite) of distinct subspaces $M$ in $\mathscr{F}$ for which $\alpha_{M}=\alpha$.

Propostrion 2. Let $M \in \mathscr{F}$ and let $\delta>0$ be given. Then there exists a subspace $L$ in $\mathscr{F}$ such that $L \subset M$ and for every $p$ in the basis $P$ of all continuous seminorms on $X$ we have

$$
\tilde{p}_{L}([T x]) \leqslant \delta \quad\left(x \in M_{-}\right)
$$

where $[y]$ denotes the coset $y+L(y \in X)$ and

$$
\tilde{p}_{L}([x])=\inf \{p(y): y \in[x]\} .
$$

Remark. The interest of this lemma lies in the case in which $M=M_{-}$. When $M \neq M_{-}$, the result is trivial since we may take $L=M_{-}$.

Proof. Suppose that the lemma is false, and denote by $\mathscr{F}_{0}$ the class of all $L$ in $\mathscr{F}_{F}$ such that $L \subset M$. Since we are going to vary $L$ we shall not use the notation $[y]$ for cosets, but throughout the proof will write $y+L$. If $L \in \mathscr{F}_{0}$, the set

$$
S_{L}=\left\{x \in U \cap M_{-}: \tilde{p}_{L}(T x+L)>\delta \text {, for some } p \text { in } P\right\}
$$


is not empty. Since $S_{L} \subseteq S_{N}$ if $N \subseteq L$ the family $\left\{S_{L}: L \in \mathscr{F}_{0}\right\}$ forms a filter base on the set $U$. Hence the family $\left\{T S_{L}: L \in \mathscr{F}_{0}\right\}$ forms a filter base on the precompact set $T U$. This filter has at least one accumulation point in the compact set $K$ formed by taking the closure of $T U$ in the completion $\hat{X}$ of $X$. Since

$$
\tilde{p}_{L}(y+L)>\delta \quad\left(y \in T S_{L}\right)
$$

it follows that

$$
\tilde{p}_{L}\left(x_{0}+L\right) \geqslant \delta
$$

where we use the same notation for the extension of $p$ to $\hat{X}$. Furthermore we have $S_{L} \subseteq M, T S_{L} \subseteq M_{-}$and hence

$$
x_{0} \in \mathrm{cl}\left[\bigcup\left\{L: L \in \mathscr{F}_{0}\right\}\right],
$$

the closure being taken in the completion of $X$. Thus for some $L$ in $\mathscr{F}_{0}$, we may choose an element $y$ in $L$ such that $p\left(x_{0}-y\right)<\delta$. This contradicts (2) and the lemma is proved.

Proposition 3. Let $\rho$ be a non-zero eigenvalue of $T$ and $x$ a corresponding eigenvector. Let

$$
M=\bigcap\{L: L \in \mathscr{F}, x \in L\} .
$$

Then $M \in \mathscr{F}$ and $\rho=\alpha_{M}$.

Proof. The property (ii) of simple nests immediately implies that $M \in \mathscr{F}$. In proving that $\rho=\alpha_{M}$ we shall consider separately the two cases in which (respectively) $M=M_{-}$and $M \neq M_{-}$.

(a) Suppose that $M=M_{-}$. Choose $\delta$ so that

$$
0<\delta<|\rho|
$$

and let $L$ be chosen to satisfy the conclusions of Proposition 2. Since $L \subset M$ and $L \in \mathscr{F}$ it is an immediate consequence of the definition of $M$ that $x \notin L$. We may choose, by a corollary to the Hahn-Banach theorem, a continuous linear functional $f$ on $M$ such that $f(x)=1$ and $f(y)=O(y \in L)$. Let $p$ be the seminorm defined by

$$
p(z)=|f(z)| \quad(z \in M) .
$$

Define

$$
d(u, L)=\inf _{z \in L} p(u-z) \quad(u \in M)
$$

Observe that if $y \in L$, then $T y \in L$ and hence

$$
\begin{aligned}
|\rho| & =|\rho| d(x, L)=d(\rho x, L)=d(T x, L) \\
& =d(T x+T y, L) \leqslant \delta .
\end{aligned}
$$

The last inequality follows from Proposition 2. This contradicts (3). Hence case (a) cannot occur. 
(b) We may now suppose that $M \neq M_{-}$. Then $x \in M$, but $x \notin M$, since $M$ is, by definition, the smallest member of $\mathscr{F}_{p}$ containing $x$. Let $z_{M} \in M \backslash M_{-}$, and let $y_{M}$ in $M_{-}$be chosen so that $T z_{M}=\alpha_{M} z_{M}+y_{M}$. We may put

$$
x=\beta z_{M}+y,
$$

where $y \in M_{-}$and $\beta \neq 0$. Then

$$
\begin{aligned}
0 & =T x-\rho x=T\left(\beta z_{M}+y\right)-\rho\left(\beta z_{M}+y\right) \\
& =\beta\left(\alpha_{M} z_{M}+y_{M}\right)+T y-\rho\left(\beta z_{M}+y\right) \\
& =\beta\left(\alpha_{M}-\rho\right) z_{M}+\beta y_{M}+T y-\rho y
\end{aligned}
$$

Now $y, y_{M}$ and (since $M_{-}$is invariant under $T$ ) $T y$ are all elements of $M_{-}$but $z_{M} \notin M_{-}$. Hence $\beta\left(\alpha_{m}-\rho\right)=0$, and since $\beta \neq 0$, it follows that $\alpha_{M}=\rho$.

Proposition 3 asserts that a non-zero eigenvalue of $T$ is a diagonal coefficient of $T$. We now prove a result in the opposite direction.

Proposition 4. Let $M \in \mathscr{F}$ and suppose that $\alpha_{M} \neq 0$. Then $\alpha_{M}$ is an eigenvalue of $T$.

Proof. It is sufficient to show that $\alpha_{M}$ is an eigenvalue of the operator $T$ obtained by restricting $T$ to the space $M$. Since $\alpha_{M} \neq 0$ we have $M \neq M_{-}$. Now $T_{M}$ is completely continuous. From equation (1) it follows that the range of the operator $T_{M}-\alpha_{M} I$ is contained in $M_{-}$, and is therefore not the whole space $M$. It follows from Theorem $A$ that $\alpha_{M}$ is an eigenvalue of $T_{M}$ and hence of $T$.

Proposition 5. Let $\rho$ be a non-zero eigenvalue of $T$. Then the diagonal multiplicity of $\rho$ is equal to its algebraic multiplicity as an eigenvalue of $T$.

Proof. Let $d$ denote the diagonal multiplicity, $m$ the algebraic multiplicity, and $\nu$ the index of $\rho$ relative to $T$. Then

(a) $\nu$ is the least integer such that $(T-\rho I)^{\nu+1} x=0$ only if

$$
(T-\rho I)^{\nu} x=0 \quad(x \in X)
$$

(b) $\nu$ is the least integer such that

$$
(T-\rho I)^{\nu+1} X=(T-\rho I)^{\nu} X
$$

(c) the null-space of the operator $(T-\rho I)^{\nu}$ has dimension $m$.

Let $S$ be the completely continuous linear operator defined by

$$
S-\lambda I=(T-\rho I)^{\nu},
$$

where $\lambda=-(-\rho)^{\nu}$. Then $\lambda$ is an eigenvalue of $S$ which has index unity and algebraic multiplicity $m$. Since $S$ is a polynomial in $T$, each subspace $M$ in $\mathscr{F}$ is invariant under $S$. We may therefore consider the diagonal coefficients of $S$ with respect to the nest $\mathscr{F}$.

Let $M \in \mathscr{F}$ and let $\alpha_{M}, \sigma_{M}$ denote the diagonal coefficient at $M$ of $T, S$ respectively. If $M=M_{-}$, we have $\alpha_{M}=\sigma_{M}=0$. If $M \neq M_{-}$, then with the usual notation we may deduce 
from the equation $T z_{M}=\alpha_{M} z_{M}+y_{M}$ that

$$
(T-\rho I) z_{M}=\left(\alpha_{M}-\rho\right) z_{M}+y_{M} .
$$

It easily follows that, for $n=1,2, \ldots$, we have

$$
(T-\rho I)^{n} z_{M}=\left(\alpha_{M}-\rho\right)^{n} z_{M}+y^{(n)},
$$

where $y^{(n)} \in M_{\ldots}$. In particular, by taking $n=\nu$, we obtain

$$
S z_{M}=\lambda z_{M}+\left(\alpha_{M}-\rho\right)^{\nu} z_{M}+y^{(\nu)} \text {. }
$$

Thus $\sigma_{M}=\lambda+\left(\alpha_{M}-\rho\right)^{\nu}$. We deduce that $\sigma_{M}=\lambda$ if and only if $\alpha_{M}=\rho$. Hence the diagonal multiplicity of $\lambda$ relative to $S$ is $d$. It is now sufficient to prove the lemma under the additional hypothesis that $\rho$ has index unity relative to $T$, since in the general case we may reduce to this situation by replacing $T, \rho$ by $S, \lambda$ respectively.

Suppose therefore that $\rho$ has index unity relative to $T$, and let $N$ be the null-space of the operator $T-\rho I$. Given $x \in N$, define

$$
M(x)=\bigcap\{L: L \in \mathscr{F}, x \in L\} .
$$

From Proposition 3 and its proof we deduce that $M(x) \in \mathscr{F}, x \in M(x) \backslash M_{-}(x)$ and $\alpha_{M(x)}=$ $\rho(x \in N, x \neq 0)$. The remainder of the proof is divided into three stages.

First, we show conversely that if $M \in \mathscr{F}$ and $\alpha_{M}=\rho$ then $M=M(x)$ for some non-zero $x$ in $N$. For this purpose, let $T_{M}$ denote the restriction of $T$ to $M$, and let $W_{M}$, $N_{M}$ be the range and null-space respectively of the operator $T_{M}-\rho I_{M}$. Then $T_{M}$ is a completely continuous linear operator on $M$, and it is immediate from the definition of index in terms of null-spaces that $\rho$ has index unity relative to $T_{M}$. Hence, by Theorem $A$,

$$
W_{M} \oplus N_{M}=M
$$

Since, as in the proof of Proposition $4, W_{M} \subseteq M$, it follows that $N_{M}$ meets $M \backslash M_{-}$. If $x \in N_{M} \cap\left(M \backslash M_{-}\right)$, it is easily verified that $x \in N, x \neq 0$ and $M(x)=M$.

Secondly, let $M_{1} \subset M_{2} \subset \ldots \subset M_{d}$ be distinct members of the nest $\mathscr{F}$ at which $T$ has diagonal coefficient $\rho$. We may choose non-zero vectors $x_{1}, \ldots, x_{d} \in N$ such that $M_{i}=$ $M\left(x_{i}\right)(i=1, \ldots, d)$. For each $i=1, \ldots, d, x_{i}$ is not a linear combination of $x_{1}, \ldots, x_{i-1}$; for this would imply that $x_{i} \in M\left(x_{i-1}\right) \subseteq M_{-}\left(x_{i}\right)$, which is not so. Hence, $x_{1}, \ldots, x_{d}$ are linearly independent elements of $N$, and since $\operatorname{dim} N=m$ we have $m \geq d$.

Thirdly, suppose that $m>d$. By the Hahn-Banach theorem, we can find linear functionals continuous on $X$, such that $\phi_{i}\left(x_{i}\right) \neq 0$, but $\phi_{i}(x)=0\left(x \in M_{-}\left(x_{i}\right)\right)$. Then if $x \in M\left(x_{i}\right)$ and $\phi_{i}(x)=0$, we have $x \in M_{-}\left(x_{i}\right)$. Now since $\operatorname{dim} N>d$, we may choose a non-zero vector $x$ in $N$ such that $\phi_{i}(x)=0(i=1, \ldots, d)$. Then $\alpha_{M(x)}=\rho$, and therefore $M(x)=M\left(x_{i}\right)$ for some $i$. Thus $x \in M\left(x_{i}\right), \phi_{i}(x)=0$, and we have $x \in M_{-}\left(x_{i}\right)=M_{-}(x)$. However, this is impossible. Hence $m \leqslant d$. Since the reverse inequality has already been established we have $m=d$, and Proposition 4 is proved.

We now state a theorem which summarizes the principal results obtained in the preceding lemmas. 
THEOREM 2. Let $T$ be a completely continuous linear operator on a locally convex Hausdorff topological vector space $X$ over the complex field and let $\mathscr{F}$ be a simple nest of subspaces of $X$, each of which is invariant under $T$. Then

(i) a non-zero scalar $\rho$ is an eigenvalue of $T$ if and only if it is a diagonal coefficient of $T$;

(ii) the diagonal multiplicity of $\rho$ is equal to its algebraic multiplicity as an eigenvalue of $T$;

(iii) the operator $T$ has no non-zero eigenvalue if and only if $\alpha_{M}=0(M \in \mathscr{F})$, or equivalently if and only if $T M \subseteq M_{-}(M \in \mathscr{F})$.

Proof. The only statement not already proved is (iii). From (i), it follows that $T$ has no non-zero eigenvalue if and only if $\alpha_{M}=0(M \in \mathscr{F})$.

COROLLARY. If there is a continuous simple nest of subspaces of $X$, each of which is invariant under $T$, then $T$ has no non-zero eigenvalue.

Proof. This follows from part (iii) of the preceding theorem.

ACKNOWLEDGEMENT. I should like to take this opportunity to express my gratitude to Dr. H. R. Dowson for much valuable advice and encouragement during three years of supervision at the University of Glasgow.

\section{REFERENCES}

1. M. Altman, On linear functional equations in locally convex linear topological spaces, Studia Math. 13 (1953), 194-207.

2. M. Altman, Invariant subspaces of completely continuous operators in locally convex topological spaces, Studia Math. 15 (1956), 129-130.

3. J. R. Ringrose, Superdiagonal forms for compact linear operators, Proc. London Math. Soc. (3) 12 (1962), 367-384.

4. A. P. Robertson and W. J. Robertson, Topological vector spaces (Cambridge University Press, 1964).

5. F. Treves, Topological vector spaces, distributions and kernels (Academic Press, 1967).

\section{AKTI KoYNTOYRIOTI}

Kastella

Piraeus

GrEecE 